\section{It's not all in the DNA}

Genetic parallels between organisms are often described by the similarity of their genome sequences, but this view might not be the full story. A study of histone modifications, carried out on an unprecedented scale, shows that epigenetic modifications between human and mouse often show striking conservation even when the underlying DNA sequence is not itself notably conserved.

How the genome functions depends not only on the DNA sequence but on how its higher-order structure is modulated: each 146-bp stretch of DNA in the eukaryotic genome is wrapped around a core set of eight histone molecules, which can be modified post-translationally to influence gene transcription, and thereby development, cell-cycle progression and disease. But just how does the pattern of histone modification correlate with gene activity, and is the pattern similar across species and, indeed, across the same genome? Although several narrower studies have hinted that the distribution of histone modifications differs between humans and other model systems, these questions could only be looked at more closely by surveying a substantial portion of the genome. With this in mind, a new study was carried out with two goals: to assay the degree of modification of histone $\mathrm{H} 3$ across the non-repetitive regions of human chromosomes 21 and 22, and at selected gene loci of the human genome that have orthologues in mouse.

Histones can be modified in several ways, but this work concentrated on three types: di- or tri-methylation of Lys4 on histone H3, or the acetylation on this histone of Lys9 and Lys14. The chromosome-wide epigenetic profile was created by using a sensitive and unbiased protocol in which modified DNA was isolated by chromatin immunoprecipitation; the sequences that were pulled out were hybridized to an array of overlapping nucleotide sequences to determine which sequences were overrepresented in the chromatin immunoprecipitated sample and establish the length of the modified sequence. The punctate but generally uniform distribution of histone modifications across the two chromosomes was informative: for more than 300 genes, tri-methylated Lys4 sites and acetylated sites were largely associated with the transcription start sites of active genes, whereas di-methylated Lys4 sites were located in the vicinity of start sites, but did not coincide with them.
But is there any significance in the histone enrichment of certain parts of the genome? If so, we would expect homologous sequences in closely related species to contain histones that are modified in a similar way. Indeed, the di-methylation pattern of Lys4 is conserved across two sets of mouse-human orthologous genes. What was striking, however, was that the DNA sequence of functionally conserved sequences, as inferred from histone profiling, did not coincide with higher levels of sequence conservation, implying that comparative genomics is no reliable way of detecting stretches of DNA with conserved epigenetic marks.

Spanning $39 \mathrm{Mb}$ of DNA, this is the most extensive survey of epigenetic patterns ever performed. Although the goals of the work were largely met, some puzzling questions remain: the punctate methylation patterns are not seen in Hox genes, where Lys4 dimethylated sites are broadly distributed across several genes. One large-scale study begets another.

Tanita Casci

\section{6) References and links} ORIGINAL RESEARCH PAPER Bernstein, B. E., Kamal, M. et al. Genomic maps and comparative analysis of histone modifications in human and mouse. Cell 120, 169-181 (2005)

\section{FURTHER READING Li, E. Chromatin}

modification and epigenetic reprogramming in mammalian development. Nature Rev. Genet. $\mathbf{3}$ 662-673 (2002)

\section{WEB SITES}

Stuart Schreiber's laboratory:

http://www-schreiber.chem. harvard.edu Eric Lander's laboratory:

http://web.mit.edu/biology/www/facultyareas/ facresearch/lander.shtml

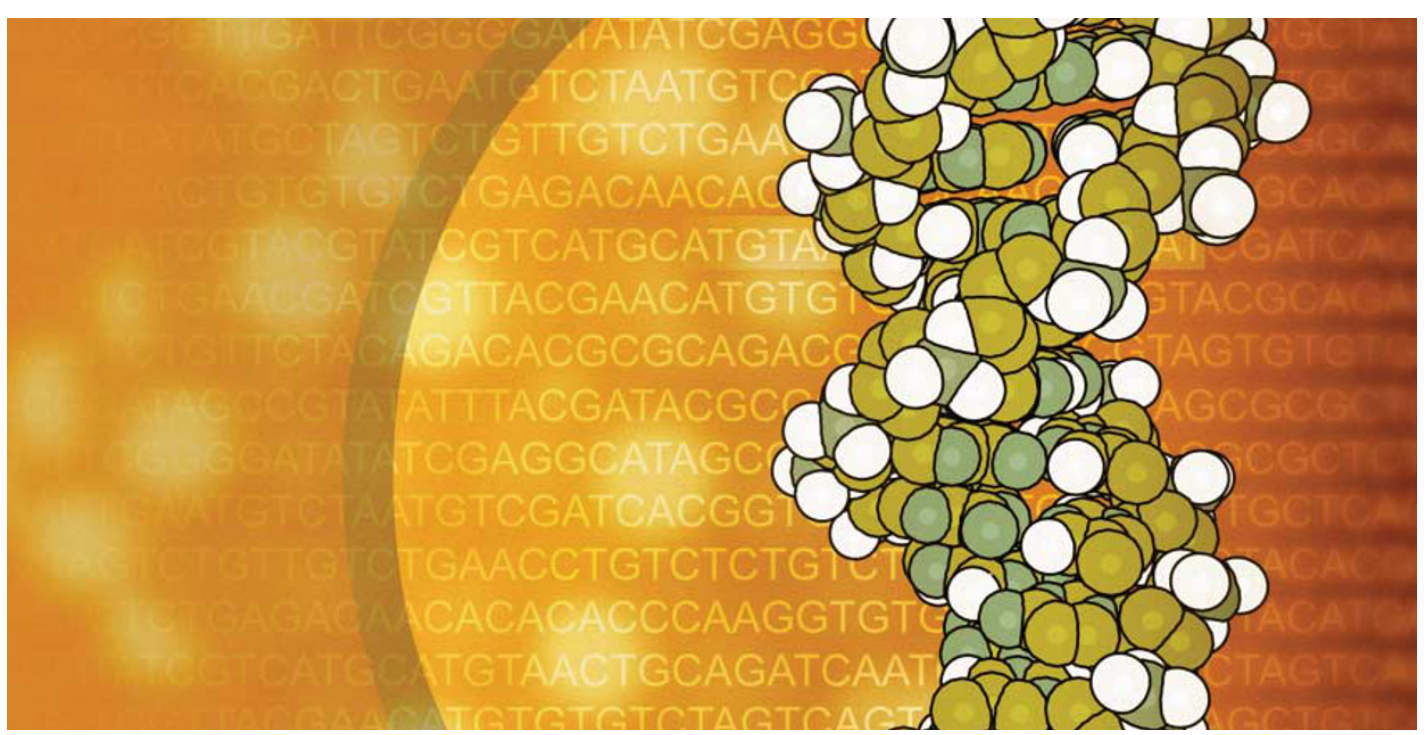

\section{IN THE NEWS}

Glow-in-the-dark smallpox Never mind GM soybeans or tomatoes! Smallpox virus, "one of the biggest killers in the history of infectious diseases" (The Independent, 22 January 2005) could soon join the list of GM organisms, that is if the World Health Organisation (WHO) committee give their approval.

This rather surprising recommendation to create GM smallpox virus comes from senior scientific advisors of the WHO and has caused quite a stir within the scientific community.

Smallpox was eradicated in 1977, but amidst fears of its use in a bioterrorist attack, there is pressure to develop new effective drugs against the disease. Nonetheless, "the man [Professor Donald Henderson] who led the successful global vaccination campaign to eradicate smallpox from the wild said he opposed the move on the grounds that the scientific benefits were not worth the risks to public health" (The Independent). He was reported to fear that a more virulent form of the virus could inadvertently be created. This scenario is not without precedent - this is exactly what happened four years ago when a team of Australian scientists modified a mousepox virus. "The researchers were trying to create a rodent contraceptive. Instead, they produced a disease that killed every

one of its mouse victims, by wiping out part of their immune system." (BBC Online, 2 February 2005)

Who knows if the fears are justified; the modification in question only involves inserting a GFP sequence into the viral genome to facilitate future drug screening. "The quickest way to screen a large database of compounds is to have an automated way" (The Independent), something that could be achieved by screening for GFP expression.

Magdalena Skipper 